\title{
Time of the Energy Emission in the Hydrogen Atom and Its Electrodynamical Background
}

\author{
Stanisław Olszewski \\ Institute of Physical Chemistry, Polish Academy of Sciences, Warsaw, Poland \\ Email: olsz@ichf.edu.pl
}

How to cite this paper: Olszewski, S. (2016) Time of the Energy Emission in the Hydrogen Atom and Its Electrodynamical Background. Journal of Modern Physics, 7, 1725-1737.

http://dx.doi.org/10.4236/jmp.2016.713155

Received: August 18, 2016

Accepted: September 25, 2016

Published: September 28, 2016

Copyright $\odot 2016$ by author and Scientific Research Publishing Inc.

This work is licensed under the Creative Commons Attribution International License (CC BY 4.0).

http://creativecommons.org/licenses/by/4.0/

(c) (†) Open Access

\begin{abstract}
The time of the energy emission between two neighbouring electron levels in the hydrogen atom has been calculated first on the basis of the quantum aspects of the Joule-Lenz law, next this time is approached with the aid of the electrodynamical parameters characteristic for the electron motion in the atom. Both methods indicate a similar result, namely that the time of emission is close to the time period of the electromagnetic wave produced in course of the emission. As a by-product of calculations, the formula representing the radius of the electron microparticle is obtained from a simple combination of the expressions for the Bohr magnetic moment and a quantum of the magnetic flux.
\end{abstract}

\section{Keywords}

Energy Emission in the Hydrogen Atom, Time of the Electron Transition between Two Quantum Levels, Electrodynamical Parameters Characteristic for the Electron Transition

\section{Introduction}

In physics, we often look for a simple explanation of the important phenomena without going much into details of the examined process. A well known example is the energy spectrum of the hydrogen atom. The first step to approach this spectrum theoretically was based on the idea that the force of the electrostatic attraction existent between the electron particle and the atomic nucleus remained in an equilibrium with the centrifugal force due to the circular electron motion about the same nucleus [1]. The second decisive step was that the angular momentum which accompanied the motion leading to the equilibrium of the atomic system should be quantized in a proper way. A combination of these two steps gave a spectacular success of the Bohr atomic model expressed in 
terms of positions of the electron energy levels present in the atom.

But the point not examined in the Bohr theory was the transition time between different quantum levels necessary to obtain the energy spectrum effect. A kind of paradox becomes that if we have two quantum states in the atom, say a hydrogen atom, their energies are well known, and the same knowledge applies naturally to the energy difference

$$
\Delta E=E_{n+1}-E_{n}>0
$$

between the states $n+1$ and $n$ entering the transition process, but we cannot answer how long is the time interval $\Delta t$ necessary to perform such transition. Certainly, the interval $\Delta t$ is classified as "short" but no quasi-definite answer on its size is in practice available.

A reason of such situation cannot be the so-called uncertainty principle between the intervals of energy and time introduced by Heisenberg [2] [3]. In fact, this principle concerns rather a mutual relation between two definite and accessible intervals $\Delta E$ and $\Delta t$ entering a given quantum process than an "uncertainty" of the accuracy which can be attained in the measurement of the sizes of the mentioned intervals [4]-[6]. This implies that there does not exist an a priori difficulty to obtain $\Delta t$ when $\Delta E$ is known.

The main source of difficulty to calculate $\Delta t$ seems to be a probabilistic- and statistical character of examination applied in the treatment of the electron transitions. This kind of approach, being typical for the old quantum theory [7] [8], obtained its farther background in the formalism of quantum mechanics [3] [9] [10]. In effect the results for $\Delta t$ connected with the electron transition obtained respectively by the classical and quantum-mechanical approaches became diametrically different [10]. For, in order to obtain an agreement with the transition intensity of energy provided by the quantum-mechanical theory, the classical approach to that intensity required the time interval $\Delta t$ of an infinite size, viz.

$$
\Delta t \rightarrow \infty \text {, }
$$

instead of a finite (small) $\Delta t$ dictated evidently by an experimental practice.

In a set of papers [11]-[15], we tried to approach the size of $\Delta t$ with the aid of an examination of the electron transitions in small quantum systems with the aid of the Joule-Lenz law; see e.g. [16] [17]. For transitions connected with the population change of the neighbouring energy levels, i.e. $n+1$ and $n$, the main result was that the relation

$$
\Delta E \Delta t=h
$$

should be satisfied. In the case of the harmonic oscillator

$$
\Delta E=\hbar \omega=h v
$$

where

$$
v=\frac{1}{T_{\text {osc }}}
$$

is the oscillator frequency of the emitted electromagnetic wave and $T_{\text {osc }}$ is the time period of that wave. In effect we obtain with the aid of (3)-(5): 


$$
h v \Delta t=\frac{h}{T_{\text {osc }}} \Delta t=h
$$

or

$$
\Delta t=T_{\mathrm{osc}}
$$

Because of (3) the emission intensity of a single wave having the frequency $v$ becomes

$$
\frac{\Delta E}{\Delta t}=\frac{(\Delta E)^{2}}{h}=\frac{(\hbar \omega)^{2}}{h}=\frac{h \omega^{2}}{(2 \pi)^{2}} .
$$

The intensity (8) can be referred to the the quantum-mechanical expression for the transition intensity of the harmonic oscillator [10]

$$
\frac{\mathrm{d} E}{\mathrm{~d} t}=\frac{2}{3} \frac{e^{2} \omega^{3} n \hbar}{c^{3} m}
$$

by the formula

$$
\frac{\mathrm{d} E}{\mathrm{~d} t}=\frac{\Delta E}{\Delta t} \gamma \frac{2 \pi}{\omega} n=\frac{\Delta E}{\Delta t} \gamma T_{\mathrm{osc}} n
$$

where

$$
\gamma=\frac{2}{3} \frac{e^{2} \omega^{2}}{m c^{3}}
$$

is the damping term of the oscillator and $T_{\mathrm{osc}}$ is the time period presented in (5); see also [10].

\section{The Aim of the Paper}

The aim of the present paper is to examine in some detail the transition time $\Delta t$ between the neighbouring quantum levels of the hydrogen atom. Certainly the size of $\Delta t$, because of its expected very short duration, seems to be hardly possible to be compared accurately with the experimental data. Nevertheless, an idea how $\Delta t$ can be influenced by the electrodynamical parameters responsible for the electron transition could be given. This makes, in principle, the problem of the time transfer $\Delta t$ between two quantum states reduced to a semiclassical one, so it can be treated with the aid of the classical electrodynamics. Before the electrodynamical properties will be discussed it seems of use to get an insight into the quantum aspects of $\Delta t$ based on the Joule-Lenz law.

\section{Electron Transition Time Obtained from the Joule-Lenz Law}

A preliminary approach to the time transfer of energy in the hydrogen atom, but not only in such system, can be done with the aid of a quantum insight into the Joule-Lenz law; see [11]-[15]. The dissipation rate of the energy $\Delta E$ within the time interval $\Delta t$ can be expressed by the formula

$$
\frac{\Delta E}{\Delta t}=R i^{2}
$$


Here the $R$ and $i$ are respectively the resistance and current intensity of the electron transition process done in course of $\Delta t$. The process leads to the energy dispense (decrease) equal to $\Delta E$.

For an unmodified (stationary) electron motion in the atom the current intensity is a constant

$$
i_{n}=\frac{e}{T_{n}}
$$

for any quantum state $n$ because of a constant time period $T_{n}$ representing the circulation of the electron charge $e$ about the nucleus [18]:

$$
T_{n}=\frac{2 \pi n^{3} \hbar^{3}}{m e^{4}} .
$$

Certainly in course of the transition from one orbit, say that of the quantum state $n+$ 1 , to the orbit of state $n$, the intensity is modified from $i_{n+1}$ to $i_{n}$, but in fact the change of $i$ is not large, especially when $n \gg 1$. Therefore, we can assume that

$$
i=i_{n+1}=\frac{e}{T_{n+1}} \approx i_{n}=\frac{e}{T_{n}} .
$$

The potential $V$ entering the electric resistance

$$
R=\frac{V}{i}
$$

let be

$$
V=V_{n}=\frac{\Delta E}{e}
$$

where in the numerator is substituted the energy difference between quantum states presented in (1). For large $n$, the energy $\Delta E$ becomes [18]:

$$
\Delta E=E_{n+1}-E_{n}=\frac{m e^{4}}{2 \hbar^{2}}\left[\frac{1}{n^{2}}-\frac{1}{(n+1)^{2}}\right] \cong \frac{m e^{4}}{\hbar^{2} n^{3}} .
$$

By combining $V_{n}$ in (17) with $i_{n}$ calculated from (13) and (14), we obtain the resistance

$$
R=\frac{\Delta E}{e i_{n}}=\frac{m e^{4}}{\hbar^{2} n^{3}} \frac{1}{e^{2}} \frac{2 \pi n^{3} \hbar^{3}}{m e^{4}}=\frac{2 \pi \hbar}{e^{2}}=\frac{h}{e^{2}}
$$

independent of the state $n$. The resistance $R$ obtained in (19) is characteristic for the integer quantum Hall effect [19]. The whole fraction (12) becomes

$$
\frac{\Delta E}{\Delta t}=\frac{h}{e^{2}} i_{n}^{2}=\frac{h}{e^{2}} \frac{e^{2}}{\left(2 \pi n^{3} \hbar^{3}\right)^{2}} m^{2} e^{8}=\frac{h}{e^{2}} \frac{e^{10} m^{2}}{(2 \pi)^{2} n^{6} \hbar^{6}}=\frac{m e^{4}}{\hbar^{2} n^{3}} \frac{1}{\Delta t}
$$

where in the last step we applied for $\Delta E$ the result of (18) obtained from large $n$.

In effect, $\Delta t$ attains the value

$$
\Delta t=\frac{m e^{4}}{\hbar^{2} n^{3}} \frac{2 \pi}{e^{8} \hbar} \frac{\hbar^{6} n^{6}}{m^{2}}=\frac{2 \pi \hbar^{3} n^{3}}{e^{4} m}
$$


which is formally equal to the size of the time period $T_{n}$ given in (14).

In the next step, by multiplying the both sides of (21) by $\Delta E$ taken from the final step of (18) we obtain

$$
\Delta E \Delta t=\frac{m e^{4}}{\hbar^{2} n^{3}} \frac{2 \pi \hbar^{3} n^{3}}{m e^{4}}=2 \pi \hbar=h .
$$

This is a result providing us with a very simple relation between the $\Delta E$ and $\Delta t$.

The relation identical with (3) has been rather extensively applied in comparing the quantum-mechanical spectrum of transition probabilities between the electron states in the hydrogen atom [20] [21] with the intensity of the electron transitions calculated with the aid of $\Delta E$ and $\Delta t$ entering the formula (22), i.e. the formula

$$
\frac{\Delta E}{\Delta t}=\frac{(\Delta E)^{2}}{h}
$$

similar to (8) for the oscillator has been applied; see [11]-[15]. More precisely, the formula (23) is valid solely for transitions

$$
n+1 \rightarrow n
$$

but the transition time $\Delta t$ corresponding, say, to situations

$$
\begin{aligned}
& n+2 \rightarrow n, \\
& n+3 \rightarrow n, \\
& n+4 \rightarrow n,
\end{aligned}
$$

etc, can be composed from the $\Delta t$ calculated for the case of (23) [13] [14].

Another important point concerning $\Delta t$ in (23) is its reference to the time period $T$ of the electromagnetic wave produced by the energy difference $\Delta E$. In fact because of the result

$$
\Delta t=T_{n}
$$

obtained in (21), the formula (23) becomes reduced to

$$
\frac{1}{\Delta t}=\frac{\Delta E}{h}=v=\frac{1}{T}
$$

therefore we obtain

$$
\Delta t=T=T_{n}
$$

which is similar to (7) for the harmonic oscillator.

In fact, the formulae (23) and (27) are not specific solely for the hydrogen atom and the harmonic oscillator, but their validity can be extended to other quantum systems, for example the particle in a one-dimensional potential box; see [11] [12] [15]. Because of (21) and (27), we obtain also

$$
\frac{h}{T_{n}}=\frac{h}{T}=h v=\Delta E .
$$

In fact on the basis of of (14), we have

$$
\frac{h}{T_{n}}=\frac{h m e^{4}}{2 \pi n^{3} \hbar^{3}}=\frac{m e^{4}}{n^{3} \hbar^{2}}=\Delta E .
$$


In the last step of (27b) the result obtained in (18) has been taken into account.

The aim of the remainder of the paper is to show-for the hydrogen atom taken as an example-that the result of (26) obtained mainly on a quantum footing-could find its correspondence also in effect of a semiclassical approach to the electron transition.

\section{Electrodynamical Parameters Connected with the Electron Transition and Its Current}

First of the necessary parameters will be the magnetic flux $\Phi$ and its changes in the atom due to the changes of the population of the quantum levels [15]. The magnetic field $B_{n}$ connected with the quantum state $n$ is an effect of the electron circulation along the orbit $n$. This implies that $T_{n}$ in (14) and the time period causing the existence of $B_{n}$ should be equal. Therefore (see e.g. [22] [23])

$$
\Omega_{n}=\frac{2 \pi}{T_{n}}=\frac{e B_{n}}{m c}
$$

where the field $B$ is directed normally to the orbit plane.

A substitution of $T_{n}$ from (14) into (28) gives the relation

$$
2 \pi\left(\frac{2 \pi n^{3} \hbar^{3}}{m e^{4}}\right)^{-1}=\frac{m e^{4}}{n^{3} \hbar^{3}}=\frac{e B_{n}}{m c}
$$

from which

$$
B_{n}=\frac{m^{2} e^{3} c}{n^{3} \hbar^{3}} .
$$

The magnetic flux across the area of a circular orbit having the radius [18]

$$
r_{n}=\frac{n^{2} \hbar^{2}}{m e^{2}}
$$

is equal to

$$
\Phi_{n}=B_{n} S_{n}=\frac{m^{2} e^{3} c}{n^{3} \hbar^{3}} \pi \frac{n^{4} \hbar^{4}}{m^{2} e^{4}}=\pi \frac{\hbar c n}{e}=\frac{h c n}{2 e}
$$

because the area enclosed by the orbit amounts to

$$
S_{n}=\pi r_{n}^{2}=\pi\left(\frac{n^{2} \hbar^{2}}{m e^{2}}\right)^{2}=\pi \frac{n^{4} \hbar^{4}}{m^{2} e^{4}},
$$

on condition the radius $r_{n}$ in (31) is taken into account.

Evidently the absolute change of $\Phi_{n}$ associated with the change of $n$ by

$$
|\Delta n|=1
$$

provides us for any $n$ with the value

$$
\Delta \Phi=\frac{h c}{2 e} .
$$

This is a quantum a) independent of $n, b)$ well-known from the physics of supeconductors [23]-[25]. Let us note that if 


$$
\mu_{B}=\frac{e \hbar}{2 m c}
$$

is the Bohr magneton [3], the formula (35a) multiplied by $2 \pi$ and divided by the flux in (35) gives

$$
\frac{2 \pi \mu_{B}}{\Delta \Phi}=2 \pi \frac{e \hbar}{2 m c} \frac{2 e}{h c}=\frac{e^{2}}{m c^{2}}
$$

which is a distance known as the radius of the electron microparticle; see e.g. [17].

In fact the steady orbital current $i_{n+1}$ is perturbed in course of transition from $n+1$ to $n$, nevertheless we expect this perturbation is small. The effective current of transition let be

$$
i_{t r}=i_{n}+i_{d}(t)
$$

where solely

$$
i_{d}(t) \ll i_{n}
$$

is the current part dependent on time $t$. Assuming that the orbits system between states $n+1$ and $n$ behaves like a condenser, our idea is to introduce a current

$$
\frac{\mathrm{d} e}{\mathrm{~d} t}=i_{d}
$$

representing a discharge of the condenser [17] [26]. This

$$
i_{d}(t)=i_{d}
$$

enters (36) and (37).

The interval $\Delta \Phi$ in (35) is coupled with the self-induction constant $L$ by the formula

$$
\frac{1}{c} \Delta \Phi=L i_{t r}=L\left(i+i_{d}\right)
$$

but the differentiation process with respect to time concerns solely the term $i_{d}$.

$$
\frac{1}{c} \frac{\mathrm{d}}{\mathrm{d} t} \Delta \Phi=L \frac{\mathrm{di} i_{d}}{\mathrm{~d} t} .
$$

The resistance $R$ is

$$
R=\frac{V}{i}=\frac{\Delta E}{e i}
$$

and the capacitance $C$ for a planar condenser is

$$
C=\frac{e}{V}=\frac{e^{2}}{\Delta E} .
$$

But because of a cylindrical shape of the orbits forming the condenser the formula (36) should be replaced by [26]

$$
C=\frac{2 e^{2}}{\Delta E}
$$

The $L, R$ and $C$ parameters enter the time-dependent differential equation for the 
current $i_{d}$ in (36) (see [17]):

$$
L \frac{\mathrm{d}^{2} i_{d}}{\mathrm{~d} t^{2}}+R \frac{\mathrm{di} i_{d}}{\mathrm{~d} t}+\frac{1}{C} i_{d}=\frac{\mathrm{d}}{\mathrm{d} t} \mathcal{E}_{\text {appl }}
$$

where $\mathcal{E}_{\text {appl }}$ is the applied electromotive force. If we assume that

$$
\mathcal{E}_{\text {appl }}=\mathcal{E}_{\text {oappl }} \mathrm{e}^{-j \omega t},
$$

where

$$
j=(-1)^{1 / 2},
$$

the solution of (45) becomes

$$
i_{d}=i_{d_{0}} \mathrm{e}^{-j \omega t} ;
$$

the $e$ in (46) and (48) is the basis of the natural logarithms.

On condition we assume that

$$
\mathcal{E}_{\text {appl }}=0,
$$

the differential process of (45) performed upon (48) gives

$$
\left(-L \omega^{2}-R j \omega+\frac{1}{C}\right) i_{d}=0 .
$$

The solution of (50) leads to the frequency [17] [26]:

$$
\omega=-j \frac{R}{2 L} \pm \sqrt{\frac{1}{L C}-\left(\frac{R}{2 L}\right)^{2}} .
$$

\section{Calculation of the Frequency $\omega$}

From Equation (40), we have

$$
L \cong \frac{\Delta \Phi}{c i}=\frac{h}{2 e i}
$$

because of (35), so in view of (44):

$$
L C=\frac{\Delta \Phi}{c i} \frac{2 e^{2}}{\Delta E}=\frac{h}{2 e i} \frac{2 e^{2}}{\Delta E}=\frac{e h}{\Delta E i} .
$$

In the next step from (42) and (52)

$$
\frac{R}{2 L}=\frac{1}{2} \frac{\Delta E}{e i} \frac{2 e i}{h}=\frac{\Delta E}{h} .
$$

Therefore, (51) becomes

$$
\omega=-j \frac{\Delta E}{h} \pm \sqrt{\frac{i \Delta E}{e h}-\left(\frac{\Delta E}{h}\right)^{2}}=-j \frac{\Delta E}{h} \pm \sqrt{\frac{e}{T} \frac{\Delta E}{e h}-\left(\frac{\Delta E}{h}\right)^{2}} .
$$

Since

$$
h v=\frac{h}{T}=\Delta E
$$

we have 


$$
\frac{1}{T}=\frac{\Delta E}{h},
$$

so the expression entering the square root in (55) is equal to

$$
\frac{e \Delta E}{T e h}-\left(\frac{\Delta E}{h}\right)^{2}=0
$$

In effect $\omega$ in (51) becomes equal to the imaginary value

$$
\omega=-j \frac{\Delta E}{h}=-j \frac{1}{T} .
$$

This result gives for the current in (48) the formula

$$
i_{d}=i_{d_{0}} \mathrm{e}^{-j \omega t}=i_{d_{0}} \mathrm{e}^{-\frac{t}{T}},
$$

With $e$ in (60) being the basis of natural logarithms, we obtain for $i_{d}$ a current exponentially decreasing with time $t$.

Let us note that when $R=0$, which is the case where no resistance does exist for the transition current, the expression for $\omega$ becomes [see (51)]:

$$
\left.\omega\right|_{R=0}=\sqrt{\frac{1}{L C}}=\sqrt{\frac{\Delta E i}{e h}}=\sqrt{\frac{\Delta E}{T h}}=\frac{\Delta E}{h}=\frac{1}{T}=v
$$

in virtue of (57).

\section{Emission Rate and Its Damping Time}

On the basis of (20) and (21), it is easy to calculate the emission rate

$$
\frac{\Delta E}{\Delta t}=\frac{m e^{4}}{\hbar^{2} n^{3}} \frac{e^{4} m}{2 \pi \hbar^{3} n^{3}}=\frac{m^{2} e^{8}}{2 \pi \hbar^{5} n^{6}} .
$$

By substituting for simplicity $m \approx \hbar \approx 10^{-27}$ and $e \approx 4.8 \times 10^{-10}$, we obtain

$$
\frac{\Delta E}{\Delta t} \cong \frac{\left(10^{-27}\right)^{2-5}}{2 \pi n^{6}}\left(4.8 \times 10^{-10}\right)^{8} \approx \frac{10^{6} \times 2.8}{2 \pi n^{6}} \mathrm{erg} / \mathrm{sec} .
$$

This is a very high number especially for small $n$, nevertheless it is valid solely at the very beginning of the emission process. The duration of that process for the energy interval $\Delta E$ is approximately equal to [see (21)]:

$$
\Delta t=\frac{\Delta E}{\Delta E / \Delta t}=\frac{2 \pi \hbar^{3} n^{3}}{e^{4} m} \cong \frac{2 \pi \times\left(10^{-27}\right)^{3-1} n^{3}}{\left(4.8 \times 10^{-10}\right)^{4}} \approx \frac{2 \pi n^{3}}{5.3} 10^{-16} \mathrm{sec} .
$$

\section{Velocity of the Electron Transition between Two Neighbouring Quantum Levels}

The result of (21) and (26) allows us to calculate the velocity of transition of the electron particle between the levels $n+1$ and $n$. This is

$$
v_{t r}=\frac{\Delta r_{n}}{\Delta t}=\frac{\Delta r_{n}}{T_{n}}=\frac{\Delta E}{h} \Delta r_{n},
$$


where [see (31)]

$$
\Delta r_{n}=\frac{(n+1)^{2}-n^{2}}{m e^{2}} \hbar^{2}
$$

With $\Delta E$ represented by a difference in the kinetic electron energy

$$
\Delta E=-\Delta E_{\text {kin }}=\frac{m}{2}\left(v_{n}^{2}-v_{n+1}^{2}\right)=\frac{m}{2}\left(\frac{e^{2}}{\hbar}\right)^{2}\left[\frac{1}{n^{2}}-\frac{1}{(n+1)^{2}}\right],
$$

this gives

$$
v_{t r}=\frac{e^{4}}{h \hbar^{2}} \frac{m}{2} \frac{(n+1)^{2}-n^{2}}{n^{2}(n+1)^{2}} \frac{(n+1)^{2}-n^{2}}{m e^{2}} \hbar^{2} \cong \frac{e^{2}}{h} \frac{1}{2} \frac{(2 n)^{2}}{n^{4}}=\frac{e^{2}}{\hbar} \frac{1}{\pi n^{2}}=v_{n} \frac{1}{\pi n} .
$$

We find that the transition velocity of the electron between levels $n+1$ and $n$ is by a factor of $1 / \pi n$ smaller than the velocity $v_{n}$ along the orbit $n$. This calculation is fully original and new.

\section{Comments}

Heisenberg strongly criticized the Bohr atomic model as useless because it applied the unobserved elements of the atomic structure like the electron orbits; see e.g. [26]-[28].

Nevertheless the combined orbital parameters, like the orbit radius or orbit length and the time period of the electron circulation, allowed us to approach correctly the parts of the electron kinetic and potential energy which — when added together-gave a proper total electron energy in the atom. This energy formula has been next confirmed by both the modern quantum theory (quantum mechanics) and experiment.

But the modern theory did not provide us with an adequate information on the time duration of the electron processes in the atom, for example the time of the electron transitions. In this circumstance, a step towards the old quantum theory which applied definite periods of time seemed to be both realistic and useful.

In the first step, we assumed that the classical Joule-Lenz theory can couple the amount of energy emitted in the quantum process of an electron transition with the time necessary for that process. This assumption led to an extremely simple relation between the emitted energy $\Delta E$ and emission time $\Delta t$. In the present paper our aim was to approach the time necessary for the emission of $\Delta E$ on a somewhat different way than a direct application of the Joule-Lenz law, i.e. mainly with the aid of a classical analysis of the electric current produced as an effect of transition giving the energy change $\Delta E$.

In the first step of this analysis, the quanta of the magnetic induction and magnetic flux are introduced to the formalism. It should be noted that the Bohr magneton divided by the quantum change of the magnetic flux between the neighbouring levels [see (35b)] gives the well-known formula for the radius of the electron microparticle; see e.g. [17]. An earlier derivation of (35b) - different only in a constant factor-has been done in [29]; see also [30].

Next the electric current connected with the transition between two neighbouring 
quantum levels is considered as due to a discharge of the condenser. In order to examine this current, the costants of self-induction, resistance and capacitance characteristic for such condenser have been calculated. In effect an exponential decrease of the time-dependent part of the discharge current is obtained; see (60).

A very simple approach to the discharge current from state $n$ can be attained when the Ohm's law is applied [21]:

$$
i_{\text {on }}=\frac{Q}{R C}=-\frac{\mathrm{d} Q}{\mathrm{~d} t} .
$$

Here $Q=e$ and $-\mathrm{d} Q=\mathrm{d} e$ is a small decrease of charge of the condenser in a small time interval $\mathrm{d} t$. The formula (68) gives

$$
\frac{\mathrm{d} Q}{Q}=-(R C)^{-1} \mathrm{~d} t
$$

Therefore

$$
\log Q=-\frac{t}{R C}+\text { cont }=-\frac{t}{2 T}+\text { cont }
$$

because

$$
R C=\frac{\Delta E}{e i} \frac{2 e^{2}}{\Delta E}=\frac{2 e}{i}=2 T .
$$

Hence the charge $Q$ decreases with $t$ according to the formula

$$
Q=\mathrm{e}^{-\frac{t}{2 T}} \text {. }
$$

The exponent of the natural logarithm basis e in (72) is a half of that obtained in (60). The rate of the emission in the form of the electromagnetic field energy has been discussed in [31].

It can be noted that Equations (3) and (22) are formally similar to the inequality proposed by Heisenberg called the uncertainty principle for energy and time. In fact the physical background for the intervals $\Delta E$ and $\Delta t$ entering he Heisenberg principle is much different than the properties of the intervals $\Delta p_{x}$ and $\Delta x$, concerning-for example-the $x$-coordinates of the momentum and position of a particle. For, contrary to the momenum and position, the energy $E$ can be measured to any degree of accuracy at any instant of time. Therefore $\Delta E$ can be the difference between two exactly measured values of energy at two different instants; see [4]-[6].

\section{Conclusions}

The paper approaches a seldom discussed problem of an individual electron transition between two quantum levels in the hydrogen atom. Consequently, no reference has been done to the well-known probabilistic theory usually applied to the quantum transitions.

In the first step, the emission time of energy between two neighbouring levels in the atom is calculated on the basis of the quantum aspects of the Joule-Lenz law; see (21) and (22). This time is found equal to the oscillation period of the electromagnetic wave 
emitted in course of the transition process; see (27).

Next, the problem of the emission was approached with the aid of the classical electrodynamics by assuming that the electron transition in the atom was roughly equivalent to a discharge of an electrical condenser. The damping time of the current obtained in course of such discharge is found to be close to the transition time attained in the Joule-Lenz theory; see (60) and (61), as well as (72) for the case of a simplified treatment of the calculation.

By assuming that the transition time between the quantum levels is similar to the emission time, the velocity of transition of the electron particle between the neighbouring orbits in the atom has been estimated; see (67).

A by-product of the calculations is the result that the Bohr magneton divided by the quantum of the magnetic flux obtained from the flux difference of two energy levels in the atom approaches the geometrical radius attributed to the electron microparticle; see (35a) and (35b).

\section{References}

[1] Bohr, N. (1913) Philosophical Magazine, 26, 1, 476, 827.

[2] Heisenberg, W. (1927) Zeitschrift fuer Physik, 43, 172-198. http://dx.doi.org/10.1007/BF01397280

[3] Schiff, L.I. (1968) Quantum Mechanics. 3rd Edition, McGraw-Hill, New York.

[4] Landau, L. and Peierls, R. (1931) Zeitschrift fuer Physik, 69, 56-69. http://dx.doi.org/10.1007/BF01391513

[5] Jammer, M. (1974) The Philosophy of Quantum Mechanics. Wiley, New York.

[6] Schommers, W. (1989) Space-Time and Quantum Phenomena, in "Quantum Theory and Pictures of Reality" ed. by W. Schommers. Springer, Berlin. http://dx.doi.org/10.1007/978-3-642-95570-9 5

[7] Planck, M. (1910) Acht Vorlesungen ueber theoretische Physik. S. Hirzel, Leipzig 1910.

[8] Einstein, A. (1917) Physikalische Zeitschrift, 18, 121.

[9] Slater, J.C. (1960) Quantum Theory of the Atomic Structure. Vol. 1, McGraw-Hill, New York.

[10] Mizushima, M. (1970) Quantum Mechanics of Atomic Spectra and Atomic Structure. Benjamin, New York.

[11] Olszewski, S. (2015) Journal of Modern Physics, 6, 1277-1288. http://dx.doi.org/10.4236/jmp.2015.69133

[12] Olszewski, S. (2016) Journal of Modern Physics, 7, 162-174. http://dx.doi.org/10.4236/jmp.2016.71018

[13] Olszewski, S. (2016) Journal of Modern Physics, 7, 827-851. http://dx.doi.org/10.4236/jmp.2016.78076

[14] Olszewski, S. (2016) Journal of Modern Physics, 7, 1004-1020. http://dx.doi.org/10.4236/jmp.2016.79091

[15] Olszewski, S. (2016) Reviews in Theoretical Science, 4, 336-352. http://dx.doi.org/10.1166/rits.2016.1066

[16] Lass, H. (1950) Vector and Tensor Analysis. McGraw-Hill, New York. 
[17] Matveev, A.N. (1964) Electrodynamics and the Theory of Relativity. Izd. Wyzszaja Szkola, Moscow. (In Russian)

[18] Sommerfeld, A. (1931) Atombau und Spektrallinien. 5th Edition, Vol. 1, Vieweg, Braunschweig.

[19] MacDonald, A.H. (1989) Quantum Hall Effect: A Perspective. Kluwer, Milano.

[20] Bethe, H. (1933) Quantenmechanik der Ein- und Zwei-Elektronen Probleme. In: Geiger, H. and Scheel, K., Eds., Handbuch der Physik, Vol. 24, Part 1, Springer, Berlin.

[21] Condon, E.U. and Shortley, G.H. (1970) The Theory of Atomic Spectra. University Press, Cambridge.

[22] Slater, J.C. (1967) Quantum Theory of Molecules and Solids. Vol. 3, McGraw-Hill, New York.

[23] Kittel, C. (1987) Quantum Theory of Solids. 2nd Edition, Wiley, New York.

[24] Ziman, J.M. (1972) Principles of the Theory of Solids. 2nd Edition, University Press, Cambridge. http://dx.doi.org/10.1017/CBO9781139644075

[25] Cyrot, M. and Pavuna, D. (1992) Introduction to Superconductivity and High $T_{c}$ Materials. World Scientific, Singapore. http://dx.doi.org/10.1142/1039

[26] Jauncey, G.E.M. (1948) Modern Physics. 3rd Edition, Van Nostrand, New York.

[27] Van der Waerden, B.L. (Ed.) (1968) Discussion in "Sources of Quantum Mechanics". Dover Publications, New York.

[28] De Boer, J., Dal, E. and Ulfbeck, O. (Eds.) (1986) The Lesson of Quantum Theory. NorthHolland, Amsterdam.

[29] Weyl, H. (1923) Raum-Zeit-Materie. Springer, Berlin. http://dx.doi.org/10.1007/978-3-642-98950-6

[30] Olszewski, S. (2016) Journal of Modern Physics, 7, 1297-1303. http://dx.doi.org/10.4236/jmp.2016.711114

[31] Olszewski, S. Journal of Modern Physics, 7, 1440-1448.

\section{Submit or recommend next manuscript to SCIRP and we will provide best service for you:}

Accepting pre-submission inquiries through Email, Facebook, LinkedIn, Twitter, etc. A wide selection of journals (inclusive of 9 subjects, more than 200 journals)

Providing 24-hour high-quality service

User-friendly online submission system

Fair and swift peer-review system

Efficient typesetting and proofreading procedure

Display of the result of downloads and visits, as well as the number of cited articles Maximum dissemination of your research work

Submit your manuscript at: http://papersubmission.scirp.org/

Or contact jmp@scirp.org 